\title{
A Study on the Influence of Positive Transfer of Mother Tongue on Tibetan College Students' Foreign Language Learning
}

\author{
Zhuoma Dangzeng \\ Qinghai University for Nationalities, Xining 810000, Qinghai Province, China
}

\begin{abstract}
Nowadays, the studies on the language transfer are more concerning the impact of Chinese language on English learning. Very few of them are on the phenomenon of negative transfer of Tibetan language to the Tibetan students' English learning under the trilingual(TIbetan, Chinese and English) environment. The author had a detailed analysis to the Tibetan students' writing composition on the basis of language tranfer theories such as markedness, interlanguage hyposthesis, and etc. And figure out the main grammatical errors in their compositions through the method of Error Analysis. Contrastive Analysis is also used to have comparison among the Tibetan, Chinsed and English, and individual interview as well, the reasons for the grammatical errors are figured out. The major findings of this research are as follows: The grammatical errors emerged in the Tibetan English major students' composition writing is in many fields, which includes omission, collocation, single and plural form of noun, subject - predicate concord, negative, possessive, relative clause and inverted sentence; Here is the reason for these grammatical errors: various thinking modes, in short of comparisons among the three languages and inadequate input. The methods can be applied to resolve are: language instructors should intsruct language learners propers through having comparison between the three languages and more importantly, strengthenging the students' comprehensible and adequate input the learner langauge.
\end{abstract}

Key words: Language transfer; Chinese and English

Publication date: April, 2021; Publication online: 30 April, 2021

*Corresponding author: Zhuoma Dangzeng, 2697137327@qq.com

\section{Introduction}

The process of Tibetan students' English learning is a complicated process which involves many external and internal factors. Normally what the language research studies or SLA studies more focused is the learning strategies, learning motivation, learning attitude and etc. So the language transfer influenced by the L1 and L2 of Tibetan students' English learning is a blank space. This research tries to find the behind reasons that can account for the language transfer in this phenomenon.

With having more demand on the personnel who skills in the various language capability in our country, third language teaching and learning of minority students have become a core focus in the college education. Tibetan students who learn English as their third language is a case in the point. As is known to all, Tibetan students are under the bilingual education, in which Tibetan and Chinese language coexisting at the same time. For Tibetan students who grew up in the traditional Tibetan families usually acquired the Tibetan as their mother tongue first and learned the Chinese as their second language under the standard linguistic instruction in the school education. Subsequently, those who have access to learning the English in the early stage of their lives might have more advantages over learning the English as their major at college. Studies have shown that those who have bilingual learning experience have more priorities than those monolingual language learners.

\section{The Definition of Language Transfer}

Language transfer, known as cross-linguistic influence, refers to the influence from the similarities or the differences between the languages or the acquired language to the target language learning. Since the late 1960s, the study of language transfer has been an important topic in the field of second 
language acquisition, applied linguistics and language studies abroad. Many scholars in China have also studied and analyzed this topic. With the change of the world economic structure, international exchanges are becoming more and more frequent, and the number of people learning three languages is increasing. Therefore, the study of Trilingual Acquisition (SLA) has attracted increasing attention. Crosslanguage transfer is a common phenomenon in SLA, which has become the focus of interlingual influence research.

Whitney used the term "transfer" to refer to the crosslinguistic influence. But in the academic field, they tend to use the term "interference" proposed by the Weinreich (1953). At first, Weinreich $(1953: 1)$ used this term to refer to the deviations from the norms of any language that occur in bilinguals' speech are the result of bilinguals' familiarity with more than one language. This definition focuses on the negative transfer, because it takes the mother tongue as an interference between the the correct acquisition and form production. And in fact, mother tongue also has a positive influence on the acquisition of the target language. Consequently, the term "transfer" gradually replaced the "interference".

Terence Odlin (1989) also gave a detailed definition on the language transfer : transfer is the influence of the differences between the acquired ( may be acquired inaccurate) language and the target language. In his definition, the acquired language means the mother language. The transfer caused by the similarity between the mother language and the target language is positive transfer. If the learner applied the knowledge of the mother language inappropriately in the target language acquisition, it caused negative transfer. (p.27) This definition not only includes the positive transfer, but also the neagative transfer. For instance, the underproduction, overproduction, production errors, substitutions, calques, alterations of structures and misinterpretation and the different time or the duration for the learners who have different backgrounds of mother tongue.

However, there are other researchers found that the transfer phenomenon in the language contact is far more complicated than the classifications made by the Odlin (1989), and transfer can be found in all levels of the language : phonology, syntax, semantics and pragmatics. For this Kellerman \& Sharwood $(1986,1)$ Smith put forward a neutral theoretical concept: cross - linguistic influence, generally refers to the transfer, interference, avoidance, borrowing and the phenomenon of language attrition that related to the L2 and etc.

\section{The Features of Tibetan Language}

As a subfamily of the Sino-Tibetan family of langauges, Tibetan belongs to the Tibeto-Burman family, which is also the daily language used by six million Tibetan people. Tibetan script was created by Thume Sambota, one of the King Songtsen Gampo's important misnisters in $7^{\text {th }}$ century AD. Spoken Tibetan is different greatly in different places of Tibetan areas. While written Tibetan is all similar to the whole Tibetan areas. The Tibetan language has a history of many years. A large number of books have been written and translated in the Tibetan language in history, which has played an important role in the development of Tibetan culture and the preservation of documents. Oral Tibetan can divided into three kinds: U-stang, kham and Amdo. The differences between the three dialects are mainly reflected in the pronunciation, and the similarities and differences between them are generally as follows: some phonetic phenomena are different from U-stang, Kham and Amdo, while U-stang and Kham are both the same and slightly different. Some phonetic phenomena are different between Kham and Amdo and Ustang, while Kham and Amdo are both the same and slightly different. Generally speaking, U-stang dialect is quite different from Amdo dialect, while Kham dialect is between them.

Tibetan is the main communicative tool of the Tibetan people. In Qinghai, Gansu, Sichuan, Yunnan and other provinces, some of the splash people and Han people living together, there are also bilingual Chinese.Tibetan is a pinyin language, and there is a certain distance between written language and modern spoken language, but because Tibetan and local phonetic sound have a set of corresponding laws, people everywhere can spell. So despite the differences in dialects, Tibetan is still the common written communication tool of the Tibetan people.

Tibetan have gone through three developemental process, which is characterized in its pronunciation, grammar and writing and etc. Here the basic structural features of syntax , vocabulary and tense of Tibetan will be disscussed:

Tibetan sentences focus on hypotaixis. It means to express various meanings and relations between words by formal signs, principally by morphological changes of verbs and by using of relatives, therefore Tibetan sentences are formed formally. With verb as the head, Tibetan sentences are formed by words which are connected by various relatives. 
Tibetan is a typical language with verbs at the end of the sentence. The syntax structure of Tibetan is the structure of $\mathrm{S}+\mathrm{O}+\mathrm{V}+$ others. There are five structural types of Tibetan sentences: (1)(words representing items involved) + transitive verbs (are followed by additional items to show tense, person, etc.) (2) Performative verbs + intransitive verbs (are followed by additional items to show tense, person, etc.) (3) Nominal words + nouns + judgment verbs (equal to judgment sentences in Chinese) (4) Nouns + nouns (adjectives) + judgment verbs (equal to descriptive sentences in Chinese) (5) Nominal words + relatives + nouns + existential verb (equal to existential sentences in Chinese).

\section{The Influence from the Tibetan to English learning}

Tibetan is the first language for most Tibetan students, to whom Tibetan, Chinese and English are learned subsequently in the language learning process. Due to the time of setting up English class is very late in many Tibetan areas or sometimes even there is no English class arrangement in many rural Tibetan schools so that it makes harder for Tibetan English major students to learn English systematically as a major at college. Even those who have a high English level in previous study experience will necessarily make mistakes in word spelling, pronunciation or grammatical rules.

In Qinghai province, English teaching and learning has been a pivotal part in the foreign language learning for minority college students like Tibetans. Because Tibetan English major students has been undergoing a complicated linguistic structure influenced by the two main languages in their life ---- Tibetan, mother tongue and Chinese, their second language. Thus the research study on the bilingual influence of transfer on the Tibetan students' English learning has been an open field that can be further excavated. This study mainly talks about the influential factors of Tibetan and Chinese language transfer on the Tibetan English major students' English learning.

Due to the special learning background, the English level of the students in one class may varies from one person to the another. For example, many Tibetan English major students from the Foreign Language Department of Qinghai Nationalities University did not have English class during their high school education so that they may have different obstacles in the course of teaching and learning, such as grammar, vocabulary, tense, syntactical structure and the pattern or the way of English critical thinking, which in a way is extremely important for second language learner.

In the foreign Language Department of Qinghai Nationalities University, usually there are three kinds of teaching modes for Tibetan English major students: (1 )teach English in Tibetan (normally the English teachers are Tibetan teachers who can speak Tibetan, Chinese and English.), (2) teach English in Chinese (normally English teachers are the Chinese teachers who speak Chinese and English), (3) teach English in English (normally the foreign teachers who can only speak English).

It is of great importance to probe over the influential factors that can account for the reason why Tibetan English major students have difficulties in acquiring the English language. Due to the special cultural and social background in Tibetan society, the language they speak at home and the language they speak in the social contexts are total two different ones, for instance, almost all the Tibetan students speak mother tongue (Tibetan) at home or their daily life in remote areas, while they have to use Chinese when they are schooling at college or in the modern cities like downtown or prefectures. Thus there is a common phenomenon that under this bilingual background, different forms of language transfer, positive or negative, may occur occasionally in the process of learning the English as their third language. Under the influence of globalization and advancement of technology and economy, as the international language around the world, English has been becoming an imperative subject in the compulsory nine-year education in China. Nevertheless, this change has been triggering the difficulties for minority students to acquire the third language without fully learning the Chinese as their second language. On the other hand, language system of these three languages are total different kinds, including its word order, sentence structure, phonology, lexical components, and grammatical expressions. Especially in the course of acquiring the three languages at the same time.

The tradition and custom in Tibetan areas differs from those in Chinese main land, which in a sense is considerably influenced by the religion, traditional culture, regional differences in Tibetan areas. Many reasons can be responsible for the low efficiency of teaching and learning the English as a third language in Tibetan areas, such as low development in culture and economy, low income of those who live in remote Tibetan pastoral areas, the high dropout rate due to the large number of children in one family, low quality of the teaching equipment, poor teaching resources and not matching the textbooks in different semesters and etc. The shortage of 
cultivating trilingual talentsn in Tibetan areas is in urgent need of remedy in order to survive in the face of multi-cultural integration and the common development of multi-ethnic groups in China. The low English level of Tibetan students makes it difficult for them to get the desired job in the market and more importantly they can not comply with the future developmental tendency, and thus would directly affect the communication and cooperation with other regional or even international challenge. So it is in great need of tackling this problem immediately and the only and the most efficient way is getting these minority students trained professionally through the university education, of which foreign language teaching and learning is of great count for those minority students mentioned above.

Currently the studies on Tibetan and Chinese language transfer on the acquisition of English is an open field that needs further empirical and theoretical research either in the English Teaching Academic Field or the education research. This study is trying to filling the blank space of the Tibetan and Chinese language transfer research on English, which has a great significance in both teaching and learning the English as a third language in the Tibetan or even in other minority places in the main land of China. On the other hand, because this study covers three languages and a deep understanding and the trilingual ability is a key point in the process of having research on this study.

Tibetan students are not like those minorities living in the mainland, most of them do not have the access to involving in the Chinese-speaking language environment, thus on the other hand, they almost have to learn two languages at the same time. What hinders Tibetan student to acquire the English not only related to the mother tongue, but also has something to do the second language that they learned in the previous time before they start to learn English. Chinese language system is different from the Tibetan language. And many studies have proven that the pronunciation of Tibetan language is similar to the English so that Tibetan students learn English is much easier than Chinese students.

\section{Conclusion}

Nowadays, the studies on the language transfer are more concerning the impact of Chinese language on English learning. Very few of them are on the phenomenon of negative transfer of Tibetan language to the Tibetan students' English learning under the trilingual(TIbetan, Chinese and English) environment. The author had a detailed analysis to the Tibetan students' writing composition on the basis of language tranfer theories such as markedness, interlanguage hyposthesis, and etc. And figure out the main grammatical errors in their compositions through the method of Error Analysis. Contrastive Analysis is also used to have comparison among the Tibetan, Chinsed and English, and individual interview as well, the reasons for the grammatical errors are figured out:

The major findings of this research are as follows:

(1) The grammatical errors emerged in the Tibetan English major students' composition writing is in many fields, which includes omission, collocation, single and plural form of noun, subject - predicate concord, negative, possessive, relative clause and inverted sentence.

(2) Here is the reason for these grammatical errors: various thinking modes, in short of comparisons among the three languages and inadequate input.

(3) The methods can be applied to resolve are: language instructors should intsruct language learners propers through having comparison between the three languages and more importantly, strengthenging the students' comprehensible and adequate input the learner langauge.

As langauge learners, Tibetan students ought to form the English thinking mode, that is to say that learn English in English. The second language acquisition researchers also should make more empirical studies using the corpus in which interlanguage errors are clearly classified. For the government, they should also provide those language researchers, language leareners and instructors with many advanced teaching hardware construction and more teaching materials to improve their English learning.

\section{References}

[1] Charlotte Hoffmann. Language acquisition in two trilingual children[J]. Journal of Multilingual \& Multilingual Development, 1985

[2] Michael Clyne, Some of the things trilinguals $\mathrm{do}[\mathrm{J}]$. 1997

[3] Rod Ellis, Second Language Acquisition[M]. 1997

[4] Janose Cenoz, Brita Hufeisen, Ulrike Jessner. Cross - linguistic influence in third language acquisition: Psychological perspectives[M]. Cleveland: Multilingual Matters Ltd, 2001.

[5] Merrill Swain, Sharon Lapkin, Norman Rowen and Doug Hart, The role of mother tongue literacy in third language learning[J]. 2009 
[6] Ulrike Jessner. Metalinguistic Awareness in multilinguals: Cognitive Aspects of Third Language Learning. Language Awareness[J]. 2010

[7] He XJ.The Influence of Language Transfer on Trilingual Acquisition: A Case Study of Tibetan College Students' English Vocabulary Learning [J].Foreign Languages, 2014(1). [8] Li ZY. A Study on Tibetan Students' Metalinguistic Awareness in Third Language Acquisition [J].Journal of Central South University: Social Science Edition, 2016(1).

[9] Lian LJ, Deng XQ. A study on the influencing factors and effective generation path of trilingual acquisition: A case study of ethnic minority college students in Xinjiang [J].Language and Translation, 2017(3).

[10] Pei BQ. A Study on the Status Quo of English Education in Multi-lingual Environment in Sichuan Ethnic Minority Areas [J].Shandong Social Sciences, 2016(6).

[11] $\mathrm{Su}$ M.A study on the negative transfer of syntax in English learning of Uygur students [D].Lanzhou: Northwest Normal University, 2009. 